\section{PSICOPATOLOGIA E SAÚDE MENTAL: QUESTÕES SOBRE OS CRITÉRIOS QUE ORIENTAMAPERCEPÇÃO CLÍNICA}

\author{
Psychopathology and Mental Health: Questions on the \\ Standards which Guide Clinical Perception
}

\author{
Psicopatología y Salud Mental: Pregunta sobre los Criterios \\ para la Percepción de Clínica
}
Psychopathologie et Santé Mentale: Questions sur les Normes qui Guident la Perception Clinique

\begin{abstract}
Resumo
Esse estudo supõe que a formação do campo da saúde mental implicou mutações nos critérios de apreensão dos problemas mentais em relação à psicopatologia. Pode-se dizer que se trata de um deslocamento fundamental, qual seja, a loucura como patologia mental deixa de ser a questão central das disciplinas psiquiátricas e psicológicas, uma vez que se instaura um domínio científico que aborda o sofrimento psíquico como uma problemática relacionada à satisfação e à realização pessoal dos indivíduos. Desse modo, se propõe aqui um estudo sobre os fundamentos epistemológicos dos saberes e das práticas em psicopatologia, conforme a perspectiva de Canguilhem, que consiste na tese de que a especificidade da racionalidade médica é o caráter normativo da apreensão dos fenômenos vitais. Dessa forma, questionamse os parâmetros normativos que caracterizam a psicopatologia, mais especificamente, psiquiatria e psicanálise, orientando formas de intervenções terapêuticas dos padecimentos mentais. Ressalta-se, então, que a percepção do psicopatológico é regido pelos critérios de diferenciação entre o normal e o patológico que pressupõe a irredutibilidade patológica de certos fenômenos psíquicos. Daí procura-se indicar que os desdobramentos epistemológicos constitutivos do campo da saúde mental implicam modificações do estatuto médico dos padecimentos mentais, uma vez que perde sua especificidade em relação às doenças orgânicas; reformas das estratégias de intervenção, tecnologias e políticas sociais, as quais são dissociadas dos diagnósticos e, finalmente, mudanças dos critérios de definição do objeto clínico, qual seja, a referência à polaridade normal-patológico.
\end{abstract}

Palavras-chave: saúde menta; psicopatologia; clínica; normalidade; loucura.

\section{Abstract}

This paper relies on the assumption that the formation of the mental health field led to mutations in the criteria for apprehension of mental problems in relation to psychopathology. There is a fundamental shift, that is, madness as a mental pathology is no longer the central object of psychiatric and psychological disciplines, since a new scientific domain that approaches psychic suffering as an issue related to satisfaction and personal achievement is established. Therefore it is proposed on this paper a study on the epistemic foundations of knowledge and practice in psychopathology according to Canguilhem (1946/1995) thesis that the specificity of medical rationale is the normative basis through which vital phenomena are apprehended. Accordingly the normative parameters of psychopathology field, more specifically, psychiatry and psychoanalysis, as they determine the methods of therapeutic interventions in mental illness, are reassessed. It is noteworthy that the perception of what is psychopathological is governed by the criteria for the differentiation between what is normal and what is pathological, which implies the pathological irreducibility of certain psychic phenomena. Hence the endeavors to point out that the epistemic implications constitutive of mental health field demand modifications on the medical statute of mental illness, as it
Artigo Original
1) Professora titular do Programa de Pós-Graduação em Psicologia da Universidade de Fortaleza; Doutorado em Saúde Coletiva pelo IMS-UERJ; membro do GT-ANPEPP - Dispositivos clínicos em Saúde Mental.

2) Doutoranda em Psicologia da UNIFOR, Bolsista do Programa de Suporte à Pós-Graduação de Instituições de Ensino Particulares (Prosup/Capes), Docente do Curso de Psicologia Fanor-Devry Brasil.
Recebido em: 25/02/2012 Revisado em: 05/09/2013 Aceito em: 28/05/2014 
loses its specificity regarding organic sickness. They also require changes in the strategies of intervention, technologies and social policies as well as in the criteria for defining the clinical object, namely the normal-pathological polarity.

Keywords: mental health; psychopathology; clinical; normal; madness.

\section{Resumen}

Este documento se basa en el supuesto de que la formación del campo de la salud mental dirigido a mutaciones en los criterios para la aprehensión de los problemas mentales en relación con la psicopatología. No es un cambio fundamental, es decir, la locura como una patología mental ya no es el objeto central de las disciplinas psicológicas y psiquiátricas, ya que un dominio de los nuevos conocimientos cientificos que se acerca al sufrimiento psíquico una cuestión relacionada con la satisfacción y la realización personal se ha establecido. Por lo tanto, es este trabajo en el estudio propuesto sobre la base del conocimiento y la práctica epistémica en la psicopatología Según Canguilhem (1946/1995) la tesis de que la especificidad de la justificación médica es la base normativa a través de cual los fenómenos vitales son aprehendidos. En consecuencia, los parámetros normativos de la psicopatología de campo, más específicamente, la psiquiatría y el psicoanálisis, ya que determinan los métodos de las intervenciones terapéuticas en la enfermedad mental, se revisan. Es de destacar que la percepción de lo que es psicopatológico se rige por los criterios para la diferenciación entre lo que es normal y lo patológico, lo que implica la irreductibilidad de ciertos fenómenos psíquicos patológicos. Por lo tanto el punto en octubre a los esfuerzos que las consecuencias constitutivos del campo epistémico de la salud mental modificaciones de la demanda sobre el estatuto médico de enfermedad mental, ya que pierde especificidad excelente relativas a la enfermedad orgánica. Ta mbién se requieren cambios en las estrategias de intervención, las tecnologías y las políticas sociales, así como en los criterios de objeto que define el clínico, Es decir, la polaridad normal y lo patológico.

Palabras clave: salud mental; psicopatología; clínica; normal; locura.

\section{Résumé}

Cette étude il suppose que la formation du champ de la santé mentale a impliqué des mutations dans les critères d'appréhension des problèmes mentaux concernant à la psychopathologie. On peut dit que sur un décalage fondamental, c'est à dire, la folie comme pathologie mentale cesse d'être la question centrale des disciplines psychiatriques et psychologiques, vu que on instaure un domaine scientifique que s'approche de la suffrance psichique à la condition de problematique en rapport à la satisfation et a réalisation personnel des individus. De cette façon, la proposition ici c'est de faire une étude sur les fondements epistemológicos des savoirs et des pratiques dans psychopathologie, comme la perspective de Canguilhem (1946/1995), qu'il consiste à la thèse dont la spécificité de la rationalité médicale est le caractère normatif de l'appréhension. En conséquence, on s'interroge sur les paramètres normatifs qui caractérisent la psychopathologie, plus spécifiquement, la psychiatrie et la psychanalyse, en donnant la direction des formes d'interventions thérapeutiques des souffrances mentales. Il peut être détaché, alors, que la perception du psychopathologique est régie par les critères de différenciation entre le normal et le pathologique, lequel estime l'irréductibilité pathologique de certains phénomènes psychiques. À partir de là, il se cherche à indiquer que les dédoublages epistemológicos constitutifs $d u$ champ de la santé mentale impliquent des modifications du statut médical des souffrances mentales, vu que perd sa spécificité en rapport les maladies organiques; réformes des stratégies d'intervention, technologies et politiques sociales, qui sont dissociées des diagnostics; et, finalement, modifications de critères de définition de l'objet clinique, ce qui est, à réfèrence à la polarité normal-patologique.

Mots-clés: santé mentale; psychopathologie; clinique; normal; folie.

Este trabalho tem origem na idéia de que a atividade em saúde mental contemporânea voltada para o sofrimento psíquico implica modificações da psicopatologia no que diz respeito à definição do seu objeto e de suas funções. Isto porque se pressupõe que a referência à saúde mental como paradigma da atividade clínica envolve deslocamentos em relação ao sistema normativo, com base no qual se define o psicopatológico. Ora, os cuidados em saúde mental não são impulsionados, prioritariamente, pelos questionamentos sobre a especificidade do patológico e suas relações com o normal? Trata-se, de acordo com a tese de Castel (1987), da recomposição de um campo de conhecimento e prática que envolve mudanças, primeiramente, no estatuto médico dos problemas mentais, perdendo sua especificidade em relação às doenças orgânicas; segundo, nas estratégias de intervenção, tecnologias e políticas sociais, as quais são dissociadas dos diagnósticos e, finalmente, nos critérios de definição do objeto clínico, qual seja, a referência à polaridade normal-patológico.

Desse modo, se propõe aqui um estudo de caráter epistemológico, uma vez que se questiona o sistema normativo que caracteriza as intervenções em saúde mental, considerando o pressuposto de que houve deslocamentos em relação aos critérios clínicos da psicopatologia, implicando, assim, mudanças no estatuto clínico do sofrimento psíquico. É possível supor que é esse deslocamento dos critérios que orientam a percepção clínica que permite abordar comportamentos, anteriormente apreendidos como problemáticos somente sob a óptica das leis e dos ideais sociais (a delinquência, por exemplo), à luz de um sistema que rege a diferenciação entre saúde e doença, o qual demarca a especificidade da abordagem clínica.

Assim, indagamos sobre os critérios que orientam um 
profissional em saúde mental que toma aos seus cuidados não só indivíduos com problemas psicopatológicos psicoses e neuroses - mas sujeitos cujo modo de vida acarreta riscos à saúde física, ao desenvolvimento pessoal e ao desempenho social. Para explorar esse questionamento, podemos tomar como exemplo a obesidade, que não é em si mesma uma doença, na medida em que não se trata de uma impossibilidade orgânica, tampouco desorganização psíquica, mas é tomada aos cuidados do profissional de saúde (médico, nutricionista, psicólogo, psiquiatra) pelo fato de ser fator de risco de várias doenças orgânicas e, também, sociais, tais como a discriminação e exclusão social. Além disso, a obesidade é causa de doenças (cardíacas, por exemplo) e de fracasso social, consequentemente, de insatisfação pessoal, portanto, de sofrimento psíquico. Dessa maneira, a obesidade se torna um problema médico pelos possíveis danos físicos, pessoais e sociais que possa ocasionar e não por seu caráter patológico. Na verdade, talvez não seja incorreto dizer, em conformidade aos estudos de Castel (1987), que qualquer aspecto da vida do indivíduo constitui objeto da intervenção em saúde mental, desde que ameace o bem-estar dos indivíduos e dos grupos. Daí, a indagação de Ehrenberg (2004, p. 13): "Pode-se ainda distinguir entre infelicidades e frustrações da vida ordinária e sofrimento patológico?".

Com efeito, é possível assinalar, seguindo essa linha de raciocínio, que a instituição do risco como parâmetro de problematização das condutas dos indivíduos implica abalos consideráveis nos fundamentos teóricos e técnicos da abordagem psicopatológica dos problemas médicopsicológicos. Assim, podemos ressaltar que esses desdobramentos implicam um deslocamento importante, qual seja, a loucura como patologia mental deixa de ser a questão central das disciplinas psiquiátricas e psicológicas. Trata-se, então, de um domínio científico referente à saúde mental, que, conforme o documento constitucional da Organização Mundial de Saúde - OMS (1946), diz respeito ao estado de bem-estar e, não somente, ausência de patologia.

Por conseguinte, podemos dizer que o sofrimento psíquico se constitui em objeto da intervenção em saúde mental na medida em que o bem-estar se torna o fim por excelência das ações médicas, sobrepondo, então, a preocupação com a doença e sua terapêutica. Com efeito, todo sofrimento passa a ser digno de cuidados médicos, digam respeito aos quadros psicopatológicos específicos ou às experiências humanas cotidianas geradoras de infelicidade. Desde os tipos clínicos clássicos, como histeria, esquizofrenia, neurose obsessiva, melancolia, dentre outras, até os estados indefinidos de mal-estar ou situações existenciais complexas, como envelhecimento, separações conjugais, dificuldades no trabalho, perda de entes queridos, fracassos escolares, etc.

Com efeito, o redimensionamento do campo psicopatológico, envolvendo saberes, práticas e políticas públicas em saúde mental produzem efeitos que extrapolam o perímetro de atividade clínica, transformando as relações sociais e a forma de cuidados consigo mesmo. Como exemplo, podemos citar a importância atribuída à autonomia na sociedade individualista atual, como ideal social. Também é possível levar em conta as reformas das políticas públicas de assistência ao portador de transtornos mentais, as quais implicam alterações legais, jurídicas e institucionais.

Contudo, dentro dos limites do presente trabalho, nosso objetivo é examinar os desdobramentos das diretrizes teóricas e práticas da psicopatologia a partir das quais é possível pensar o redimensionamento dos parâmetros de apreensão dos problemas psíquicos. Em outros termos, queremos explicitar os critérios epistemológicos que sustentam a clínica psicopatológica, tendo em vista salientar os desdobramentos da percepção clínica implicados na instauração do campo de assistência em saúde mental.

\section{Questões Metodológicas}

Devemos explicitar, em primeiro lugar, que nesse estudo abordamos os saberes psicopatológicos à luz da perspectiva epistemológica de Canguilhem (1943/1995) sobre a especificidade da racionalidade médica, que consiste em considerar, conforme a análise de Serpa Junior (2006), o campo de conhecimento biológico autônomo em relação à racionalidade mecanicista de apreensão dos fenômenos físicos. O traço fundamental, conforme a tese de Canguilhem, é que um domínio de conhecimento sobre a vida implica considerar seu caráter normativo, pois a vida é uma realidade que só pode ser apreendida como movimento em oposição à morte, assim como a saúde deve ser entendida em oposição à doença, uma vez que a normatividade do organismo se opõe aos mecanismos patológicos. Portanto, nosso fio condutor no exame das disciplinas psicopatológicas, mais precisamente a psiquiatria e psicanálise, é a posição normativa que assumem em relação aos fenômenos psíquicos, uma vez que consideramos a pertinência dessas disciplinas a um campo de saberes e práticas, cuja referência essencial é o caráter de polaridade dos fenômenos vitais.

Dessa maneira, situado nesse campo de visão, partimos do princípio que definir a especificidade do psicopatológico em relação à totalidade da experiência humana é, certamente, o ponto de partida de uma disciplina médica na área de clínica psicológica. Trata-se, por um lado, de definir o psicopatológico em relação ao normal, ou seja, precisar em termos racionais, médico-científicos, em que consiste 
a doença no domínio dos transtornos psíquicos. E, de outra parte, explicar as fronteiras e as relações entre a doença e a saúde mental, uma vez que está submetida ao modelo médico, pois, como ressalta Canguilhem (1943/1995, p. 21) sobre a atividade clínica, "dominar a doença é conhecer suas relações com o estado normal que o homem vivo deseja restaurar, já que ama a vida".

Em segundo lugar, esclarecemos que nosso estudo se sustenta na tese arqueológica de Foucault (1963/1994) sobre o surgimento da medicina moderna condicionado ao paradigma da anatomopatologia. Trata-se de um campo teórico e prático no qual a problematização da vida, da doença e da morte são submetidas ao olhar inquisidor do clínico acerca das regularidades/irregularidades do organismo.

Portanto, nos propomos aqui a examinar as formulações da psiquiatria e da psicanálise, considerando sua inscrição em um domínio epistemológico cuja forma de apreensão dos fenômenos mentais é regida pela polaridade do normal e o patológico. Ou seja, abordamos essas duas disciplinas como perspectivas racionais visando explicitar suas posições normativas em relação ao sofrimento mental. Ao mesmo tempo, procuramos assinalar os possíveis pontos de mutação dos seus respectivos sistemas de normas quando se põem em discussão a problemática da saúde mental. Desse modo, as questões sobre as relações entre o normal e o patológico e a referência fundamental ao corpo como realidade da doença dirigem nossa abordagem das disciplinas a partir das quais acompanhamos a proposição de Castel $(1987$, p.67) de que a formação do campo em saúde mental implica "decomposição e recomposição inéditas da psicopatologia".

Com efeito, neste ensaio, consideramos, inicialmente, a inserção da clínica mental na seara médica mais ampla, que se caracteriza pela investigação e a terapêutica das doenças como fenômenos orgânicos, apreendidas pela diferença entre o normal e o patológico para em seguida, nos posicionamos no interior do terreno da psicopatologia, procurando explicitar os critérios normativos subjacentes às formulações acerca do fenômeno psicopatológico. Procuramos demarcar a especificidade dos projetos psicopatológicos, precisamente, psiquiatria e psicanálise, no que concerne ao paradigma anatomopatológico. Ora, mesmo reconhecendo o caráter heterogêneo da psicopatologia, que é um domínio formado por vários discursos sobre os transtornos psíquicos, divergentes em suas concepções respeitantes à dimensão da realidade em jogo na loucura, pressupomos um denominador comum às diversas disciplinas, cuja referência as enraíza no campo da psicopatologia, qual seja, o patológico como alvo de investigação e de cuidados clínicos. Por último, conjeturamos possíveis desdobramentos da psiquiatria e da psicanálise no campo de saúde mental.

É importante ressaltar que não pretendemos aqui estabelecer comparações acerca da pertinência e/ou eficácia clínica, ética e política das teorias e práticas aqui examinadas. Trata-se, precisamente, de um recorte sobre a teoria psiquiátrica e psicanalítica tendo em vista suas referências à polaridade entre o normal e o patológico e/ou à saúde mental como parâmetros da percepção clínica. Com efeito, com essas discussões referentes às especificidades epistemológicas da psicopatologia em relação à saúde mental, não pretendemos exaltar as virtudes políticas de uma clínica em saúde mental, que rompe com certos aspectos virulentos do modelo psicopatológico, tais como a separação dos indivíduos em termos de normal e patológico, justificando práticas de exclusão dos doentes mentais. Tampouco intentamos lamentar o declínio da hegemonia de uma concepção médica restrita a certo domínio da experiência humana não extensiva a todos os problemas humanos. A pretensão aqui é de explicitar, com base na perspectiva epistemológica de Canguilhem (1943/1995), o sistema normativo que fundamenta a clínica psicopatológica, acentuando suas especificidades e possíveis desdobramentos em face ao domínio da saúde mental, chamando atenção para o fato de que não se trata da mera justaposição dos dispositivos em jogo, mas de subordinações e redimensionamentos dos critérios clínicos da psicopatologia situada no campo da saúde mental.

\section{O Paradigma Anatomopatológico}

A Psicopatologia constitui um domínio teórico-clínico composto por uma miscelânea de abordagens da enfermidade mental. Com efeito, conforme indicações de Fedida e Widlöcher (1990), as formulações acerca do sofrimento psíquico provêm de vários projetos clínicos, que posicionam de forma divergentes as concepções acerca da saúde e da doença psíquica.

Há de se reconhecer, todavia, por trás dessa diversidade epistemológica, um pano de fundo com apoio no qual se delineiam as diferenças entre as várias perspectivas clínicas, qual seja, a anatomia patológica. Trata-se aí do fundamento da percepção médica à luz da qual a doença é tomada como realidade corporal, instituindo o indivíduo doente como objeto médico, conforme o espectro arqueológico de Foucault (1963/1994) sobre o surgimento da medicina moderna. Essa identidade entre doença e decomposição corporal foi estabelecida pelo jovem médico francês Françoise Xavier Bichat (1771-1802) no início do século XIX mediante a dissecação de cadáveres. Conforme os estudos de Foucault (1963/1994), nos séculos XVII e XVIII o saber médico definia-se como uma taxonomia que consistia na classificação das grandes entidades clínicas com origem nos seus sintomas. A configuração sintomática, pois, era tomada como realidade originária e alvo da intervenção médica. Daí por que devemos considerar a relação entre doença e corpo 
como o traço constitutivo e distintivo dos saberes e das práticas médicas modernas.

Ora, pode-se dizer, tomando as formulações de Canguilhem (1943/1995), que as relações entre o normal e o patológico, norteadoras da percepção clínica, se constituem como princípio de qualificação da doença e da saúde, na medida em que a anatomopatologia consiste de um corpus teórico/prático sobre o funcionamento (as regularidades) do organismo. Dessa maneira, o normal e o patológico designam o grau de funcionalidade orgânica. Impõe-se esclarecer que o critério de diferenciação das regularidades e irregularidades do funcionamento corporal é a polaridade entre vida e morte, pois é com base na oposição entre vida e morte que se classifica a doença. Daí o caráter de bipolaridade entre saúde e doença, ou seja, todo funcionamento normal implica o patológico, que implica a morte, pois, como ressalta Foucault (1963/1994, p.40) na esteira de Canguilhem (1943/1995), o "prestígio das ciências da vida, no século XIX se devem ao caráter de bipolaridade médica do normal e do patológico".

Assim, considera-se que a racionalidade anatomopatológica baliza os aspectos em torno dos quais se encontram as várias abordagens de psicopatologia. É com relação à questão sobre identidade entre doença e corpo, à diferença irredutível entre normal e patológico e à dimensão individual da doença, que os vários projetos clínicos adquirem especificidades epistemológicas. O domínio da anatomia patológica se organiza em torno da idéia de corpo doente, que é avaliado segundo sua norma de funcionamento que impossibilita uma vida saudável. Assim, entre saúde e doença há uma relação de oposição, quer dizer, o indivíduo doente é definido pelo modo patológico de funcionamento dos mecanismos físicos e mentais ao contrário de um modo de funcionamento normal. Supomos que esse sistema de definição do objeto clínico está perdendo a força na medida em que a função médica de prevenção e promoção de saúde assume posição privilegiada acarretando a subestimação da terapêutica da doença. Desse modo, a atividade médica deixa de ser restrita aos cuidados com o patológico, mas estende a atenção à qualidade de vida dos indivíduos. É com base nessas questões que formulamos a hipótese de que esse paradigma anatomopatológico está subjugado ao paradigma da saúde mental.

\section{Dispositivos Clínicos: Psicopatologia e Saúde Mental}

O espaço da psicopatologia é composto por várias perspectivas teórico e práticas acerca do psicopatológico. É lícito expressar que a referência ao corpo como realidade da doença, cujo diagnóstico é pautado pelo grau de oposição ao normal, constitui o tema crucial sob cujo domínio se posiciona os vários projetos clínicos. Assim, a psicopatologia, como um terreno circunscrito do saber médico sobre os transtornos psíquicos, caracteriza-se por uma diversidade de teorias que abordam a problemática da especificidade do psicopatológico e de suas relações com o normal. Com efeito, com base na perspectiva epistemológica de Canguilhem (1943/1995) a propósito da racionalidade clínica da patologia mental, podemos considerar diferentes níveis de discriminação do normal e do patológico. Apesar das disparidades acerca do objeto clínico, há um denominador comum a essas abordagens, qual seja, a irredutibilidade do patológico, quer dizer, a patologia é o critério de intervenção clínica dos problemas mentais.

É necessário aqui assinalar mais uma vez que o caráter de nossa proposta é situar alguns aspectos da racionalidade da psiquiatria e da psicanálise em face das referências da anatomopatologia e seu caráter de polaridade do normal/ patológico bem como os possíveis pontos de mudanças epistemológicas. Portanto, tendo em vista a demarcação dos limites do presente texto, devemos esclarecer que não pretendemos abordar as várias concepções teóricas sobre o padecimento mental, visando um estudo exaustivo do campo da psicopatologia e da saúde mental.

Então, no campo da psicopatologia, tem-se, por conseguinte, a visão nosológica e organicista da psiquiatria. Trata-se, de um lado, da descrição e taxonomia das várias formas de enfermidade mental, herança da medicina classificatória dos séculos XVII e XVIII, mediante a qual se definem e diferenciam as grandes entidades clínicas, cada uma com seu conjunto de sintomas, sua evolução, prognóstico; de outra parte, da indagação sobre a etiologia das doenças, da oposição irredutível entre normal e patológico, que marcou a atividade clínica no final do século XIX.

Essa duplicidade arqueológica entre uma medicina classificatória e uma anatomia patológica, pode estar ligada, conforme indicações de Simanke (2002), ao fato de que o organicismo situa a psiquiatria ante o problema da dualidade mente-corpo, em virtude da especificidade de seu objeto - a doença mental. Trata-se da posição desconfortável da psiquiatria entre uma medicina e uma psicologia, da qual não pode abrir mão sob o risco de desaparecer como especialidade médica. Com efeito, podemos dizer, seguindo o raciocínio de Simanke (2002), que a psiquiatria se caracteriza por um duplo dilema: de um lado, não pode aderir estritamente ao organicismo dos transtornos mentais, sob o risco de fundir-se a neurologia, por outro, não pode abrir mão do caráter ontológico de seu objeto, qual seja, o estado patológico dos fenômenos mentais, isto é, a bipolaridade dos fenômenos mentais, pois se arrisca a tornar-se uma psicologia. 
A propósito dessa posição fronteiriça desse campo de conhecimento médico entre o orgânico e o psíquico, Lacan (1987) acentua que a nosologia psiquiátrica se desenvolve, desde o final século XIX até as primeiras décadas do século $\mathrm{XX}$, com suporte numa distinção etiológica fundamental: os transtornos mentais cuja causalidade orgânica é identificável e as enfermidades em relação às quais não é possível encontrar nenhuma correlação anatômica. Foi Kraepelin, informa Lacan (1987), quem sistematizou na nosologia psiquiátrica uma oposição entre dois grandes grupos patológicos, o grupo das demências e o das psicoses, quaisquer que sejam as denominações que tiveram ao longo desse período. Um déficit mental é o princípio explicativo das afecções que caracterizam o grupo das demências, ao passo que as psicoses devem ser reconhecidas considerandose os distúrbios da personalidade. Tal diferença se baseia no critério etiológico e evolutivo da doença os quais justificam a intervenção clínica sobre as causas dos sintomas sejam elas orgânicas ou funcionais.

Numa perspectiva menos epistemológica, Castel (1987, p.80) enfatiza o peso político desses dilemas epistêmicos da psiquiatria, considerando que a instituição da doença mental, "doença, não como a outra", como objeto clínico, diz respeito, de um lado, às estratégias de modernização da medicina mental em face de sua tradição alienista, caracterizada pela instituição hospitalar, e, de outro, ao projeto de consolidação de uma área de conhecimento e prática médica autônoma em relação à medicina geral. O encargo da doença mental implica não só dispositivos institucionais e técnicos específicos, mas teorias que sustentem a originalidade dessa área médico-psicológica. Daí o campo da psicopatologia formado pela articulação de várias disciplinas em torno do padecimento mental.

Nesse terreno, a psicanálise, numa posição mais independente ao prestígio médico, desmantela, diferentemente da psiquiatria, a polaridade radical entre o normal e o patológico. Freud em $A$ interpretação de sonhos (1900/1996a) e A psicopatologia da vida cotidiana (1901/1996c) estabelece identidade entre os fenômenos psíquicos sob o argumento de que são realizações de desejo. Nessa perspectiva, as noções de normal e patológico não apontam para uma descontinuidade absoluta entre experiências psíquicas, porquanto, nos sonhos, esquecimentos corriqueiros, paralisias histéricas e delírios, por exemplo, o que está em jogo é o desejo do sujeito.

Esse relativismo não implica, entretanto, do ponto de vista freudiano, a não especificação do patológico em relação aos outros fenômenos psíquicos, pois, para Freud (1926/1996b), a inibição, por exemplo, vai-se ver, não é uma patologia, na medida em que é um fenômeno cuja referência é o desempenho do eu, e não, fundamentalmente, o conflito intrapsíquico.
Situe-se, por consequência, a questão de saber sobre a especificidade do patológico tal como Freud (1926/1996b) sustenta, considerando, precisamente, as diferenças que ele estabelece entre sintoma e inibição. Essa investigação poderia ter seguido outras direções, entretanto, consideramos que a preocupação freudiana em precisar o conceito de sintoma pela diferença da idéia de inibição vinha ao encontro de nosso interesse acerca da situação normativa dos sintomas psíquicos. Assim, podemos retomar o tema sobre as possibilidades de desdobramentos normativos da psiquiatria e da psicanálise em face das demandas em saúde mental.

Decerto é lícito dizer que a questão fundamental da idéia freudiana do psicopatológico é a referência à dimensão da linguagem. A constituição de sistemas de representações como realidade psíquica está diretamente relacionada à condição de desamparo originária da experiência humana, definida por Freud (1926/1996b) como excesso pulsional. A função psíquica consiste em conduzir à excitação pulsional, condicionando-a a uma cadeia de representação, instituindo, dessa maneira, seu caráter simbólico. A ligação entre excitação e representação marca a ruptura com o estado de desamparo absoluto, caracterizado pela irrupção de energia pura, sem representação. A simbolização implica a subordinação das ligações entre representações às leis de associações e não à de descarga, isto é, ao princípio do prazer. Nessa linha de raciocínio, o psicopatológico diz respeito a essa dimensão da experiência humana, a das pulsões e suas representações, ou seja, experiência de satisfação. Assim é com relação à tendência do aparelho psíquico à satisfação, a qual é submetida às leis associativas e seus mecanismos, que os sintomas são entendidos. Ora, estes dizem respeito aos impulsos recalcados, à realização de desejo interditada. $\mathrm{Na}$ verdade, possuem o estatuto de substituto do recalcado e, nessa condição, como anota Freud (1926/1996b), implica o rebaixamento da satisfação em compulsões.

Assim, o mecanismo de recalcamento é essencial para a especificação do patológico e aponta para aspectos tópicos, econômicos e dinâmicos do funcionamento do aparelho psíquico. Com efeito, o recalcamento é constitutivo de uma divisão subjetiva, na qual o inconsciente é a referência para a configuração do patológico. Como substituto do impulso inconsciente, o sintoma se forma por meio de um processo tortuoso e complexo de metamorfose da ideia original, mediante deslocamentos, substituições e projeções, tanto do impulso quanto do seu representante, de tal modo que se torna irreconhecível, daí o caráter interpretativo do trabalho analítico. No que diz respeito ao seu posicionamento, o sintoma é uma formação localizada fora do ego, usufruindo, como acentua Freud (1926/1996b), do privilégio da extraterritorialidade. Além disso, o sintoma não deixa de exercer uma pressão para a satisfação pulsional, obrigando 
o ego a permanecer, incansavelmente, na posição defensiva. Agora, não somente contra o impulso inconsciente, mas contra o seu substituto. Nesse jogo metapsicológico, a formação do sintoma é um processo condicionado por duas formas de atividade psíquica: de um lado, o trabalho do aparelho para escoar energia, e, de outro, o trabalho de limitação dos meios de satisfação. Com efeito, o cenário próprio da produção do sintoma é a força, o conflito, a defesa, a luta, que implica mecanismos diversos e instâncias diferentes.

Nessa perspectiva da subjetividade como espaço de luta, de desprendimento de forças, a formação do sintoma implica a transformação necessária da economia psíquica contra a satisfação de um impulso substitutivo de um desejo inconsciente cuja satisfação põe em risco o funcionamento do aparelho. A força necessária para que o ego se defenda dos impulsos inconscientes é a angústia, um sentimento de desprazer intolerável. Assim, o sintoma diz respeito à transformação do funcionamento psíquico ocasionado pela referência a um grande perigo, e o maior temor do sujeito é a castração, de sorte que o caráter patogênico da formação do sintoma consiste do jogo de forças entre desejos recalcados e a angústia que sinaliza um grande risco para o ego.

Daí a tese, estabelecida há pouco, de que Freud considera a relação de diferença entre o normal e o patológico, na medida em que o sintoma diz respeito a conflitos intrapsíquicos, e define-se como substituto do recalcado, que implica experiência-limite, angústia e um processo de deformação do desejo constitutivo do patológico. Assim, é essa relação intrínseca com uma experiência devastadora, bem como as metamorfoses associativas, econômicas e semânticas, que conferem status patológico a determinadas representações, como, por exemplo, o medo de ser mordido por cavalos, que caracteriza a neurose fóbica do pequeno Hans, caso examinado por Freud (1926/1996b), com o objetivo de especificar o sintoma em relação à inibição e à angústia. Diante da situação de um menino que se recusa a sair de casa por medo de cavalos, Freud interroga-se sobre qual seria precisamente o sintoma psíquico: o medo, a restrição do movimento ou o objeto do medo - o cavalo. Conforme as ponderações freudianas, o medo de cavalos era o substituto do recalcado e consistia da metamorfose da idéia de ser mordido por cavalo, a qual remetia aos impulsos inconscientes em relação ao pai, os impulsos hostis. Na verdade, Freud conclui o que é necessariamente sintomático nesse caso - a substituição do pai por cavalo. $\mathrm{Na}$ análise freudiana, a angústia, que caracterizou o quadro psicopatológico do pequeno Hans, diz respeito à função do ego de indicar um perigo iminente, interno ou externo, não devendo ser, portanto, tomado como sintoma. Da mesma forma, a incapacidade de sair de casa era uma inibição que o ego de Hans se impusera para evitar o medo, não sendo em si mesma patológica.

É com base nessa concepção do psicopatológico que Freud (1926/1996b) estabelece diferenças entre sintoma e inibição. Esta é um fenômeno que diz respeito às restrições das funções do ego, "como medidas de precaução ou acarretadas como resultado de um empobrecimento de energia" (p. 2834). São, pois, as limitações das funções do eu que caracterizam uma inibição. Daí as inibições de ordem sexual, social, motora, de nutrição, cognitiva, entre tantas, as quais não são em si mesmas patológicas. As inibições só assumem caráter patológico quando condicionadas ao processo defensivo do ego contra um impulso inconsciente, assim como, por exemplo, a inibição de sair de casa, presente no caso do pequeno Hans. Somente neste contexto, conforme indicações freudianas, as inibições podem ser consideradas patologias, pois complementam o sintoma. Ora, para Freud, o patológico não se define pela disfuncionalidade do eu ou pelos limites de normatividade, mas pela compulsividade de uma configuração psíquica, resultante de um processo metapsicológico de deformação de desejos inconscientes.

Agora, podemos retomar as questões acerca dos desdobramentos da psicopatologia tendo em vista sua inserção no domínio da saúde mental. Considerando dois aspectos fundamentais da mutação do campo médicopsicológico, tais como estudou Castel (1987) - a perda da originalidade da patologia mental e o redimensionamento dos critérios normativos -, talvez seja pertinente pensar na inibição como o objeto característico de uma percepção clínica direcionada por uma inquietação acerca do desempenho pessoal com vistas à qualidade de vida. A problematização da inibição pressupõe uma reordenação da hierarquização freudiana do aparelho psíquico acerca da demarcação da subjetividade. Isso quer dizer que as funções do ego, como por exemplos, os desempenhos sexuais e cognitivos, se tornam alvos de muitas atenções em detrimento das inquietações com os conflitos inconscientes. Não queremos dizer, tal como alertou Castel (1987), que a psicanálise sucumbiu diante dessa superestimação do ego, mas que se desdobrou e, portanto, deixa de ser a definição por excelência da problemática psíquica. Se esta hipótese estiver correta, diremos que a inibição psíquica, as motivações do eu, a autoestima, por exemplo, adquirem importância sob a perspectiva da saúde mental.

Essa percepção clínica que prioriza o sofrimento psíquico tendo em vista o desempenho do indivíduo e sua inserção no ambiente está relacionada diretamente aos procedimentos empíricos e pragmáticos de classificação psicopatológica, representada pelo DSM, em suas versões III e IV, os quais implicam uma mudança epistemológica em relação ao modelo da psiquiatria clássica, de acordo com Pereira (2003) e Sonenreich (2004). Trata-se de um 
sistema diagnóstico dos transtornos mentais com base nas configurações sintomáticas e não em suas causas. Dessa forma, a tendência atual da psiquiatria, sob a influência da Escola Americana, é desvencilhar-se de questões espinhosas e querelas teóricas a respeito da natureza e da etiologia da doença mental, detendo-se em bases objetivas, empíricas e operacionalizáveis, isto é, a descrição sintomática dos transtornos psíquicos. Trata-se do agrupamento de traços que definem o alvo da intervenção, como, por exemplo, o transtorno alimentar.

Dessemodo, conclui-se que a articulaçãopsicopatologia/ saúde mental supõe reorganizações, redimensionamentos, reacomodações e desdobramentos nas formulações acerca da questão psíquica, circunscrevendo o dispositivo médico/ psicológico da atualidade. Trata-se do desdobramento da percepção clínica, antes, preponderantemente limitada pela polaridade normal/patológico, e que hoje se volta, também, para as inibições, questionadas em termos de funcionamento do eu, cujo desempenho determina os modos de inserção no ambiente e condiciona o bem-estar social e a qualidade de vida, os quais são indicadores de saúde mental. Com efeito, talvez não seja incorreto dizer que a psicopatologia em sua estrutura original ocupa um espaço restrito no campo da saúde mental.

\section{Referências}

Canguilhem, G. (1995). O normal e o patológico. Rio de Janeiro: Forense Universitária. (Originalmente publicado em1943)

Castel, R. (1987). A gestão do risco: Da antipsiquiatria à pós-psicanálise. Rio de Janeiro: F. Alves.

Ehrenberg, A. (2004). Les changement de la relation normal - pathologique. Revue Esprit, 5, 133-156.

Ehrenberg, A. (2010). La société du Malaise. Paris: Odile Jacob.

Fedida, P., \& Widlocher, D. (1990). Présentation. Revue Internationale de Psychopathologie, 1, 3-4.

Foucault, M. (1994). O nascimento da clínica. Rio de Janeiro: Forense Universitária. (Originalmente publicado em1963)

Freud, S. (1996a). A Interpretação dos Sonhos. In J. Strachey (Ed.), Edição standard brasileira das obras psicológicas completas de Sigmund Freud (Vol. 5, pp. 371-648). Rio de Janeiro: Imago. (Originalmente publicado em 1900)

Freud, S. (1996b). Inibição, sintoma e angústia. In J. Strachey (Ed.), Edição standard brasileira das obras psicológicas completas de Sigmund Freud (Vol. 20, pp. 153-173). Rio de Janeiro: Imago. (Originalmente publicado em 1926).

Freud, S. (1996c). Psicopatologia da vida cotidiana. In J. Strachey (Ed.), Edição standard brasileira das obras psicológicas completas de Sigmund Freud (Vol. 6, pp. 19-272). Rio de Janeiro: Imago. (Originalmente publicado em 1901)

Lacan, J. (1987). Da psicose paranóica em suas relações com a personalidade. Rio de Janeiro: Forense Universitária.

Organização Mundial da Saúde. (1946). Constituição. New York: Author.

Pereira, M.E.(2003). ODSM-IV eo objeto da psicopatologia ou psicopatologia para quê? Recuperado de http:// www.geocities.com

Serpa, O. D., Jr. (2006). Indivíduo, organismo e doença: a atualidade de O Normal e o Patológico de Georges Canguilhem. Revista Psicologia Clínica, 15(1). Recuperado de http://www.psi.puc-rio.br/Octavio.html

Simanke, R. T. (2002). Metapsicologia lacaniana: Os anos de formação. São Paulo: Discurso.

Sonenreich, C. (2004). Introdução à clínica psiquiátrica. In P. Delgalarondo, C. Sonenreich, \& A. M. G. R. Oda (Orgs.), História da psicopatologia: Textos originais de grandes autores (pp. 109-118). São Paulo: Lemos.

\section{Endereço para correspondência:}

Clara Virgínia de Queiroz Pinheiro

Av. Washington Soares,1321, Bloco N, sala 13, Bairro

Edson Queiroz.

CEP: 60811-905 - Fortaleza-CE.

E-mail: claravirginia@unifor.br

\section{Endereço para correspondência:}

Kelly Moreira de Albuquerque

Endereço: Rua Bento Albuquerque, 1717, apto 601, Cocó

CEP: 60192-055, Fortaleza-CE.

E-mail:kellynha.psico@hotmail.com 